\title{
Análise espaço-temporal da cobertura vegetal em uma bacia hidrográfica na região do MATOPIBA, Brasil
}

\author{
Luciano Cavalcante de Jesus FRANÇA ${ }^{1 *}$, João Batista Lopes da SILVA ${ }^{2}$, Gerson dos Santos \\ LISBOA $^{3}$, Danielle Piuzana MUCIDA ${ }^{4}$, Clebson Lima CERQUEIRA ${ }^{5}$, Elyzama Lima SANTOS ${ }^{6}$
}

\begin{abstract}
${ }^{1}$ Programa de Pós-Graduação em Engenharia Florestal, Universidade Federal de Lavras - UFLA, Lavras, MG, Brasil. ${ }^{2}$ Instituto de Humanidades, Artes e Ciências, Universidade Federal do Sul da Bahia - UFSB, Teixeira de Freitas, BA, Brasil.

${ }^{3}$ Instituto de Humanidades, Artes e Ciências, Universidade Federal do Sul da Bahia - UFSB, Itabuna, BA, Brasil. ${ }^{4}$ Programa de Pós-Graduação em Ciência Florestal, Universidade Federal dos Vales do Jequitinhonha e Mucurí - UFVJM, Diamantina, MG, Brasil

${ }^{5}$ Programa de Pós-Graduação em Ciências Florestais, Universidade Federal do Paraná - UFPR, Curitiba, PR, Brasil. ${ }^{6}$ Programa de Pós-Graduação em Solos e Nutrição de Plantas, Universidade Federal do Piauí - UFPI, Bom Jesus, PI, Brasil *Email: lucianodejesus@florestal.eng.br
\end{abstract}

Recebido em agosto/2017; Aceito em agosto/2018.

RESUMO: A bacia hidrográfica do rio Uruçuí-Preto, Piauí, com área de $15.777 \mathrm{Km}^{2}$, vem caracterizando-se ao longo dos últimos 30 anos pela expansão do agronegócio, integrando a fronteira agrícola do MATOPIBA, composta por Maranhão, Tocantins, Piauí e Bahia. Este estudo objetivou avaliar a mudança da cobertura vegetal nesta bacia hidrográfica entre 1984 a 2015. Utilizou-se imagens dos sensores TM e OLI dos satélites Landsat 5 e 8, respectivamente, para elaboração de mosaicos e realce da vegetação por meio do Índice de Vegetação da Diferença Normalizada (IVDN), para obtenção da evolução da mudança na cobertura da terra. Procedeu-se a classificação supervisionada (Máxima Verossimilhança), em cinco classes: solo exposto, área antropizada, vegetação rala, vegetação esparsa e vegetação densa. Os resultados atestaram intensa antropização na área analisada. Em 1984, a classe solo exposto correspondia a $390,3 \mathrm{~km}^{2}$ do total da área da bacia, com aumento em 2015 para 1.498,20 km². Em 1984 existiam 7.743,2 $\mathrm{km}^{2}$ de cobertura vegetal original, reduzida em 2015, para $3.487,40 \mathrm{~km}^{2}$, com redução de 45,03\% da classe de vegetação densa. Este estudo pode auxiliar em estratégias de atuação dos órgãos ambientais e planejamento ambiental para o desenvolvimento do agronegócio em consonância com a conservação dos recursos naturais, sobretudo no MATOPIBA.

Palavras-chave: IVDN, desmatamento, Cerrado, sensoriamento remoto, Uruçuí-preto.

\section{Space-temporal analysis of vegetable coverage in a hydrographic basin of the region of MATOPIBA, Brazil}

\begin{abstract}
The hydrographic basin of the Uruçuí-Preto river, Piauí, with an area of $15.777 \mathrm{~km}^{2}$, has been characterized over the last 30 years by agricultural expansion, integrating the agricultural frontier of MATOPIBA, composed of the states of Maranhão, Tocantins, Piauí and Bahia. The objective of this study was to evaluate the change in the vegetation cover in this basin from 1984 to 2015 . Images of the TM and OLI sensors of the Landsat 5 and 8 satellites, respectively, were used to elaborate mosaics and vegetation enhancement through the Vegetation Index (IVDN), to obtain the evolution of the change in land cover. The supervised classification (Maximum Likelihood) was carried out in five classes: exposed soil, anthropic area, sparse vegetation, sparse vegetation and dense vegetation. The results showed intense anthropization in the analyzed area. In 1984, the exposed soil class corresponded to $390.3 \mathrm{~km}^{2}$ of the total basin area, with an increase in 2015 to $1,498.20 \mathrm{~km}^{2}$. In 1984 there were $7,743.2 \mathrm{~km}^{2}$ of original vegetation cover, reduced in 2015 , to $3,487.40 \mathrm{~km}^{2}$, with a reduction of $45.03 \%$ of the class of dense vegetation. This study can aid in strategies for the performance of state environmental agencies and environmental planning in order to reconcile the development of agribusiness with the conservation of natural resources.
\end{abstract}

Keywords: NDVI, deforestation, Cerrado, remote sensing, Uruçuí-preto.

\section{INTRODUÇÃO}

As alterações antrópicas de habitats são consideradas as principais impulsionadoras na mudança ambiental e perda de biodiversidade (LUSTIG et al., 2015), demandando dos gestores ambientais a necessidade de identificar novas maneiras de prever com rapidez e precisão a organização da paisagem florestal pós-perturbação (SAVAGE et al., 2017).

O Brasil é um dos líderes mundiais na substituição de florestas por agricultura (JÚNIOR; LIMA, 2018) e o Cerrado apresenta-se como um dos domínios mais intensamente explorado pelo crescente aumento de atividades agrícolas em grande escala e pecuária extensiva, sofrendo com isso consequências como o manejo inadequado do solo, maior vulnerabilidade aos incêndios florestais, degradação ambiental e perda da biodiversidade.

As áreas de Cerrado transformaram-se, em curto espaço de tempo, em uma das grandes áreas produtoras de grãos de soja, devido ao baixo preço das terras e pelos incentivos fiscais 
concedidos pelo governo e ao elevado preço da soja no mercado internacional (COSTA; SANTOS, 2010).

Nas últimas três décadas intensificaram-se as alterações na paisagem do Cerrado, em função da expansão do agronegócio, o que proporcionou um desenvolvimento estratégico territorial, criando e zoneando a fronteira agrícola do MATOPIBA, composta pelos estados do Tocantins, Maranhão, Piauí e Bahia. Entretanto, essa crescente ocupação e exploração dos recursos naturais potencializa os impactos ambientais negativos, que levam à degradação do domínio (DANTAS; MONTEIRO, 2011), considerado um dos 21 hosposts globais designados para conservação prioritária (MYERS et al., 2000).

Os índices de vegetação gerados a partir de dados oriundos de sensores remotos constituem uma importante ferramenta para o monitoramento de alterações naturais ou antrópicas no uso e na cobertura da terra. Desse modo, as respostas espectrais permitem distinguir a vegetação nativa das áreas com uso agropecuário, o que facilita o planejamento e direcionamento das políticas públicas e privadas relacionadas ao manejo e conservação do solo e da água (LIMA et al., 2014). Além disso, tais índices realçam o comportamento espectral da vegetação e correlacionam-se com o vigor da vegetação verde, porcentagem de cobertura do solo, atividade fotossintética e produtividade (PONZONI et al., 2015).

A utilização de técnicas e dados de sensoriamento remoto e dos Sistemas de Informações Geográficas (SIG) mostra-se uma ferramenta de grande utilidade para o desenvolvimento de estudos ambientais e para o planejamento e gestão, principalmente quando considera-se grandes extensões territoriais, uma vez que possibilita a obtenção de informações em diferentes escalas de tempo e, ou, espaço, com o investimento relativamente baixo em recursos financeiros, pessoais e logísticos. Estudos voltados ao monitoramento da mudança espaço-temporal de paisagens naturais com emprego de técnicas de sensoriamento remoto, especificamente índices de vegetação, podem ser verificados em Saito et al. (2015); ElZeiny; Effat (2017); França et al. (2017); Junior et al. (2018); Valjarevic et al. (2018); Behera et al. (2018); Wu et al. (2018); Arsanjani (2018) e Wang et al. (2018).

$\mathrm{O}$ estado do Piauí possui uma área territorial de 250.934 $\mathrm{km}^{2}$, aproximadamente 11,5 milhões de hectares são de Cerrado, 3,4 milhões de hectares área de transição e outros 3 milhões de hectares aptos para cultivos agrícolas. Estas áreas de Cerrado localizam-se, geograficamente, em distintos sítios do estado, mas preponderam na região Sudoeste e parte do extremo Sul piauiense (REYDON; MONTEIRO, 2006). A bacia hidrográfica do rio Uruçuí-Preto, tem importância ecológica e econômica direta para mais de 12 municípios da região Sudoeste do Piauí. Encontra-se em região economicamente ativa do estado do Piauí, onde concentramse os investimentos em projetos agrícolas, sobretudo no predomínio do cultivo da soja. A bacia hidrográfica faz parte do polígono conhecido como MATOPIBA, região com uma extensão de 73 milhões de hectares, sendo 66 milhões no domínio Cerrado, incluindo 337 municípios nos estados do Maranhão, Tocantins, Piauí e da Bahia (BUAINAIN et al., 2017). Em 2013, o produto interno bruto anual a preços de mercado (PIBpm) do MATOPIBA - referência 2010 - foi estimado em R $\$ 73$ bilhões, 1,4\% do brasileiro (IBGE, 2016). Esta expansão da fronteira agrícola está diretamente ligada às condições climáticas do Cerrado, como as vastas planícies e planaltos, boas condições climáticas, solos de fácil correção e mão de obra acessível (LIMA et al., 2014).

$\mathrm{Na}$ região Sul do estado do Piauí, caracterizada por ecótono (Cerrado-Caatinga), notam-se impactos ambientais sob os recursos naturais e comunidades das localidades próximas das atividades agrícolas em grande escala de produção. Neste sentido, vislumbra-se a necessidade de trabalhos e levantamentos ambientais, avaliações de impactos à biodiversidade aquática e terrestre nas áreas próximas as exploradas pela agricultura intensiva, com finalidade de acrescentar e direcionar políticas públicas de conservação, preservação e manejo sustentável dos recursos naturais e solo no domínio desta região. Nascimento e Fernandes (2017), destacam que os impactos ambientais como a escassez dos recursos hídricos, o aumento do desmatamento, os processos erosivos e manejo do solo são assuntos que estão sendo tratados com maior frequência, para se obter soluções ou medidas mitigadoras para esses problemas ambientais.

Em função da dinâmica da expansão agrícola na região Sul do Piauí e consequente desmatamento das áreas de Cerrado, é que este trabalho objetivou realizar uma análise espaçotemporal da cobertura vegetal em um período de 31 anos (1984-2015) na bacia hidrográfica do rio Uruçuí-Preto, Piauí, Brasil.

\section{MATERIAL E MÉTODOS}

A área de estudo compreende a bacia hidrográfica do rio Uruçuí-Preto, com área de drenagem total de $15.777 \mathrm{Km}^{2}$ (Leão; Monteiro, 2009), com altimetria máxima de aproximadamente $669 \mathrm{~m}$, na porção mais ao Sul da bacia hidrográfica (Figura 1). A cobertura vegetal caracteriza-se por formações vegetais do tipo ecotonal (Cerrado-Caatinga).

As cartas de cobertura vegetal foram obtidas a partir do realce da vegetação com o Índice de Vegetação da Diferença Normalizada (IVDN) através de imagens Landsat 5, sensor TM, dos anos 1984, 1999, 2000, 2005, 2010, e Landsat 8, sensor OLI, para o 2015, uma vez que o satélite anterior apresenta registro apenas até o ano de 2011. As bandas utilizadas foram referentes ao comprimento de ondas do vermelho (banda 3: 0,62-0,69 $\mu \mathrm{m}$ ) e do infravermelho próximo (banda 4: 0,78-0,90 $\mu \mathrm{m}$ ), para assim gerar uma composição colorida.

As imagens foram corrigidas atmosfericamente para reduzir os efeitos das diferentes datas de aquisição das imagens e do ângulo solar do momento da aquisição das cenas. Executou-se a correção geométrica das imagens, para remover as distorções geométricas inseridas durante o processo de imageamento.

O IVDN, utilizado para realçar a vegetação, leva em consideração as correlações existentes entre a reflectância da vegetação e do solo, tendo em vista que a vegetação possui maior reflectância na região do Infravermelho Próximo e o solo na região do vermelho. Quanto maior for a densidade da cobertura florestal, menor será a reflectância na região do visível (vermelho), e maior será a refletância na região do Infravermelho Próximo (IVP). O IVDN é obtido pela seguinte expressão:

$$
\operatorname{IVDN}=\frac{\rho \mathrm{IVP}-\rho \mathrm{V}}{\rho \mathrm{IVP}+\rho \mathrm{V}} \quad(\text { Equação } 1)
$$

em que: IVDN: Índice de Vegetação da Diferença Normalizada; $\rho$ IVP: Reflectância da banda espectral da região 
do Infravermelho Próximo (Banda 4) e $\rho V$ : Reflectância da banda espectral da região do Vermelho (Banda 3).

Após o realce da vegetação pelo IVDN, as imagens foram classificadas pelo método de classificação supervisionada (Método da Máxima Verossimilhança - MAXVER), sendo divididas em 5 (cinco) classes, conforme apresentado na Tabela 1.

Os valores do IVDN variam entre $-1 \mathrm{a}+1$, sendo que quanto mais próximo de +1 , maior a densidade da cobertura vegetal. O solo exposto ou com vegetação rala e esparsa, apresenta valores positivos, mas não próximos à +1 . Nesta situação, ocorre absorção da radiação na faixa do infravermelho próximo, justificando o baixo valor de IVDN nessas áreas (PONZONI et al., 2015).

Todos os procedimentos neste estudo foram processados no Software ArcGIS 10.2 (ESRI, 2013).
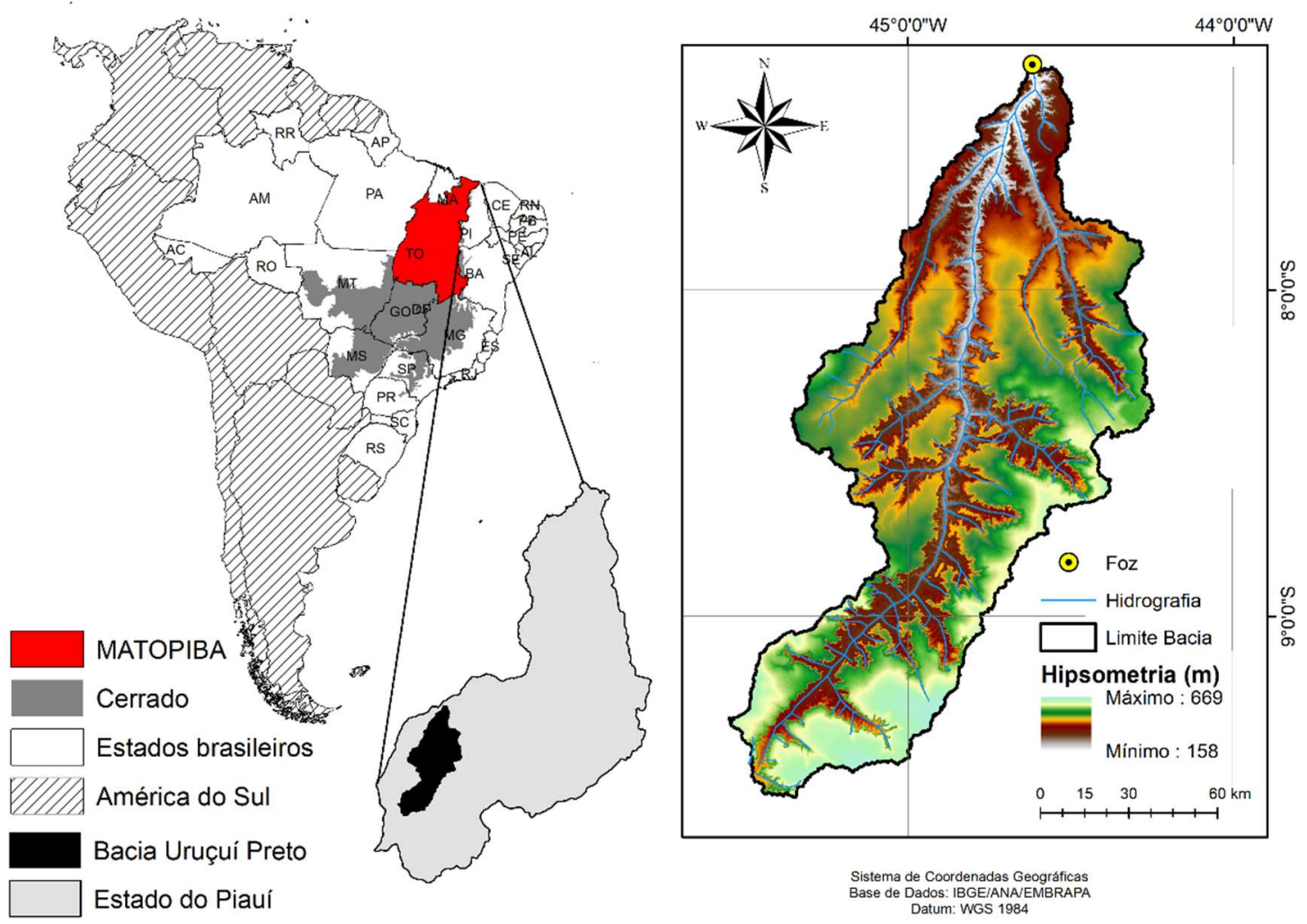

Figura 1. Mapa de Localização da Bacia Hidrográfica do Rio Uruçuí-Preto, Piauí, Brasil, com destaque para sua hipsometria. Figure 1. Location Map of the Uruçuí-Preto River Basin, Piauí, Brazil, highlighting its hypsometry.

Tabela 1. Descrição das classes de cobertura vegetal para a bacia do rio Uruçuí-Preto.

Table 1. Description of the vegetation cover classes for the Uruçuí-Preto river basin.

\begin{tabular}{cl}
\hline \multicolumn{1}{c}{ Classe } & \multicolumn{1}{c}{ Descrição } \\
\hline Solo Exposto & $\begin{array}{l}\text { Representado por sítios em que no solo não apresenta nenhum tipo de material vegetal vivo ou morto. Ou ainda } \\
\text { por áreas degradadas, afloramentos rochosos, manchas urbanas, áreas edificadas, talhões agrícolas expostos } \\
\text { (pós-colheita) ou incêndios florestais. }\end{array}$ \\
\hline Área Antropizada & $\begin{array}{l}\text { Áreas cujas características originais (solo, vegetação, relevo e regime hídrico) foram alteradas por consequência } \\
\text { de atividade humana. Caracterizam-se nesta classe os talhões agrícolas cultiváveis. }\end{array}$ \\
\hline Vegetação rala & $\begin{array}{l}\text { Vegetação em processo de sucessão ecológica (início de regeneração), ou campos naturais, típicos dos Cerrados, } \\
\text { formados por uma vegetação predominantemente baixa, com poucas árvores de troncos recurvados e de folhas } \\
\text { grossas, esparsas em meio a uma vegetação rala e rasteira. }\end{array}$ \\
\hline Vegetação Esparsa & $\begin{array}{l}\text { Podem incluir-se as formações do Cerrado típico. Característico de climas semiáridos, na faixa de transição de } \\
\text { climas úmidos para os desérticos. Tipo fisionômico exclusivamente arbustivo herbáceo, com arbustos e } \\
\text { subarbustos esparsos, cujas plantas, muitas vezes, são constituídas por indivíduos menos desenvolvidos das } \\
\text { espécies arbóreas do Cerrado sensu stricto. }\end{array}$ \\
\hline Vegetação Densa & $\begin{array}{l}\text { Remanescentes florestais, tais como Cerrado denso ou arbóreo "Cerradão", com indivíduos altos e copas } \\
\text { densas, além das zonas de Mata Ciliar/Matas de Galerias, adjacentes aos cursos d'água. }\end{array}$ \\
\hline
\end{tabular}

\section{RESULTADOS}

Os resultados do IVDN a partir das imagens Landsat TM 5 e OLI 8, para os anos 1984, 1990, 1999, 2000, 2005, 2010 e 2015 demonstraram intensa antropização na bacia hidrográfica em estudo, principalmente com a progressiva diminuição das áreas de vegetação nativa do domínio Cerrado, caracterizandose o aumento das áreas de cultivos agrícolas.

A classificação realizada no ano de 1984 apresentou os primeiros indícios do uso e ocupação da terra por atividades agrícolas (Figura 2), corroborando com Reydon; Monteiro 
(2006), os quais citam que a ocupação do Cerrado piauiense iniciou-se no início dos anos 1980 do século passado, porém, com efetiva produtividade apenas na década de 90, mediante a produção em larga escala, principalmente da soja.
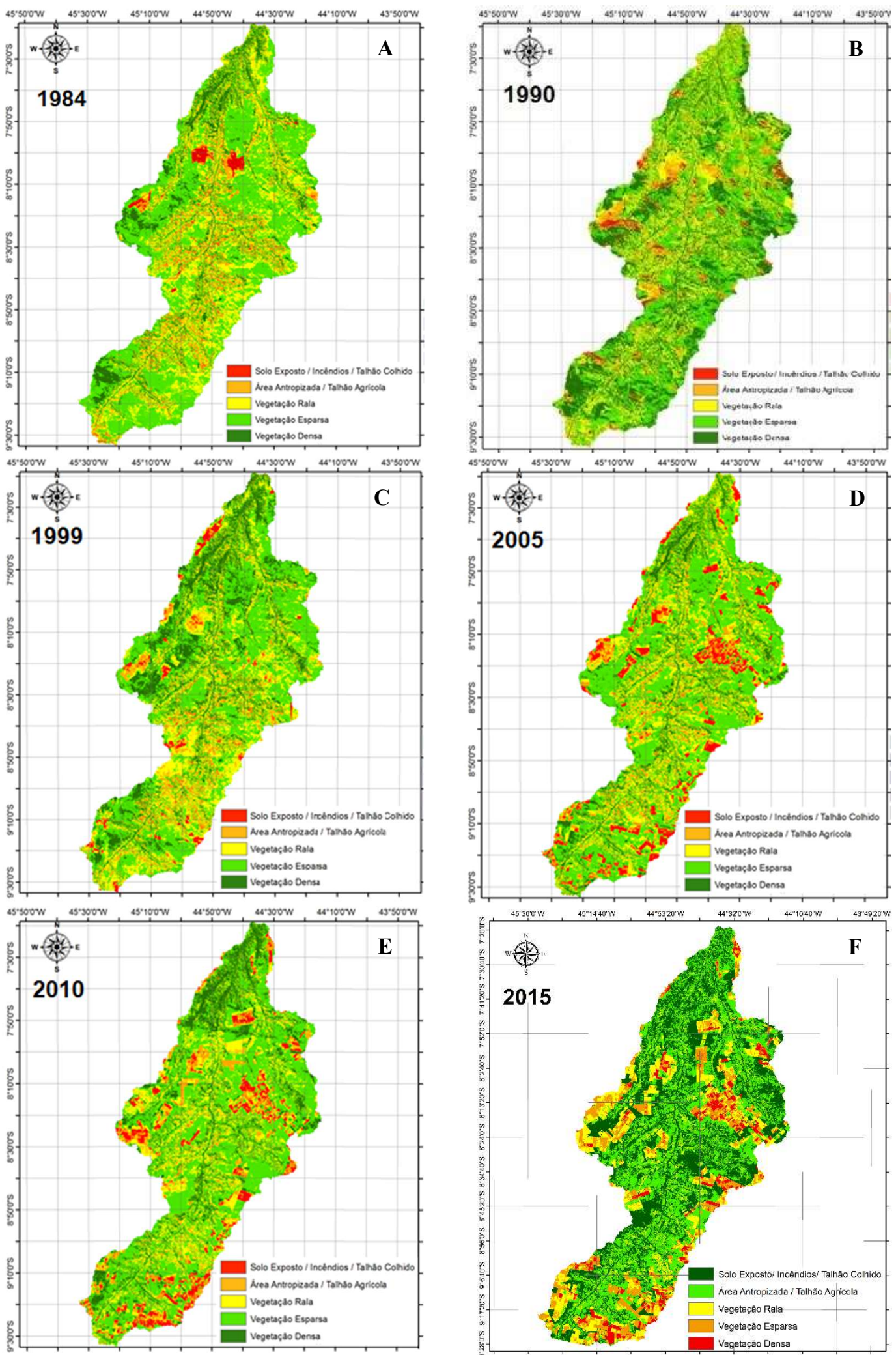

Figura 2. Distribuição das classes temáticas da cobertura vegetal da Bacia do Rio Uruçuí-Preto, nos anos de (A) 1984, (B) 1990, (C) 1999, (D) 2005, (E) 2010 e (F) 2015.

Figure 2. Distribution of the thematic classes of the vegetation cover of the Uruçuí-Preto River Basin, in the years of(A) 1984, (B) 1990, (C) 1999, (D) 2005, (E) 2010 e (F) 2015.

Na classificação realizada para o ano de 1990, constatouse suave aumento das classes de área antropizada e solo exposto (Figura 2-B), quando comparado com o ano de 1984, contudo, apresentou considerável ocorrência de incêndios 
florestais, o que é caracterizado na imagem pelas manchas sem formas definidas, diferenciando-as dos polígonos regulares dos talhões agrícolas, conforme apresentado na Figura 3. Uma das principais causas de incêndios florestais, de acordo com Medeiros (2002), é recorrente ao uso incorreto do fogo, para renovação de pastagens e limpeza de restos de culturas nas propriedades vizinhas, tratos muito frequentes nesta região do Piauí.

Nesse contexto, o aumento do risco de ocorrência dos incêndios florestais é também resultado da redução da cobertura vegetal e o aumento das atividades antrópicas, como destacam Rodrigues e Soares (2004). Sousa et al. (2012) citam que o conhecimento a respeito da estatística dos incêndios florestais é extremamente importante para implementação de estratégias de prevenção no Brasil, e que no Sul do Piauí há carência de informações dessa natureza, o que faz com que a região torne-se ainda mais vulnerável.

Analisando-se os resultados dos anos posteriores, 1999, 2005, 2010 e 2015 nas figuras 2 (C), (D), (E) e (F), respectivamente, observa-se a rápida expansão das atividades agrícolas e redução expressiva da vegetação nativa, evidenciada pelas áreas nas cores vermelho e laranja, que caracterizam a classe de solo exposto, nomeadamente as fazendas e talhões agrícolas. Já a classe representada pela cor amarela, caracteriza pela vegetação rala, o que podem ser além de áreas em regeneração natural ou a própria fitofisionomia do Cerrado Strictu Senso na concepção de Ribeiro e Walter (1998), uma vez que são formados por vegetação predominantemente baixa, com indivíduos arbóreos geralmente com troncos recurvados, esparsas em meio a uma vegetação rala e rasteira.

A mudança mais significativa foi verificada na classe de vegetação densa, caracterizada pela floresta nativa subdividida nas fitofisionomias de maior densidade do Cerrado, podendo ser representadas por: Matas Ciliares, Matas de Galerias, Mata Seca, Cerradão e Cerrado Denso (RIBEIRO; WALTER, 1998). Nesta classe, houve mudança de 7.743,2 $\mathrm{km}^{2}$ no ano de 1984, para $1.999,4 \mathrm{~km}^{2}$ em 2010, contudo, apresentando um aumento no ano de 2015, constatando-se neste ano $3.487,40 \mathrm{~km}^{2}$ de vegetação densa, o que pode estar diretamente relacionado ao período de imageamento, que nas estações de chuvas a vegetação mostra-se mais vigorosa, frente as características do domínio fitoecológico local.

As classes de área antropizada e solo exposto aumentaram progressivamente, caracterizado pelo aumento das áreas com os talhões agrícolas, conforme apresentado no Tabela 2 e Figura 3.
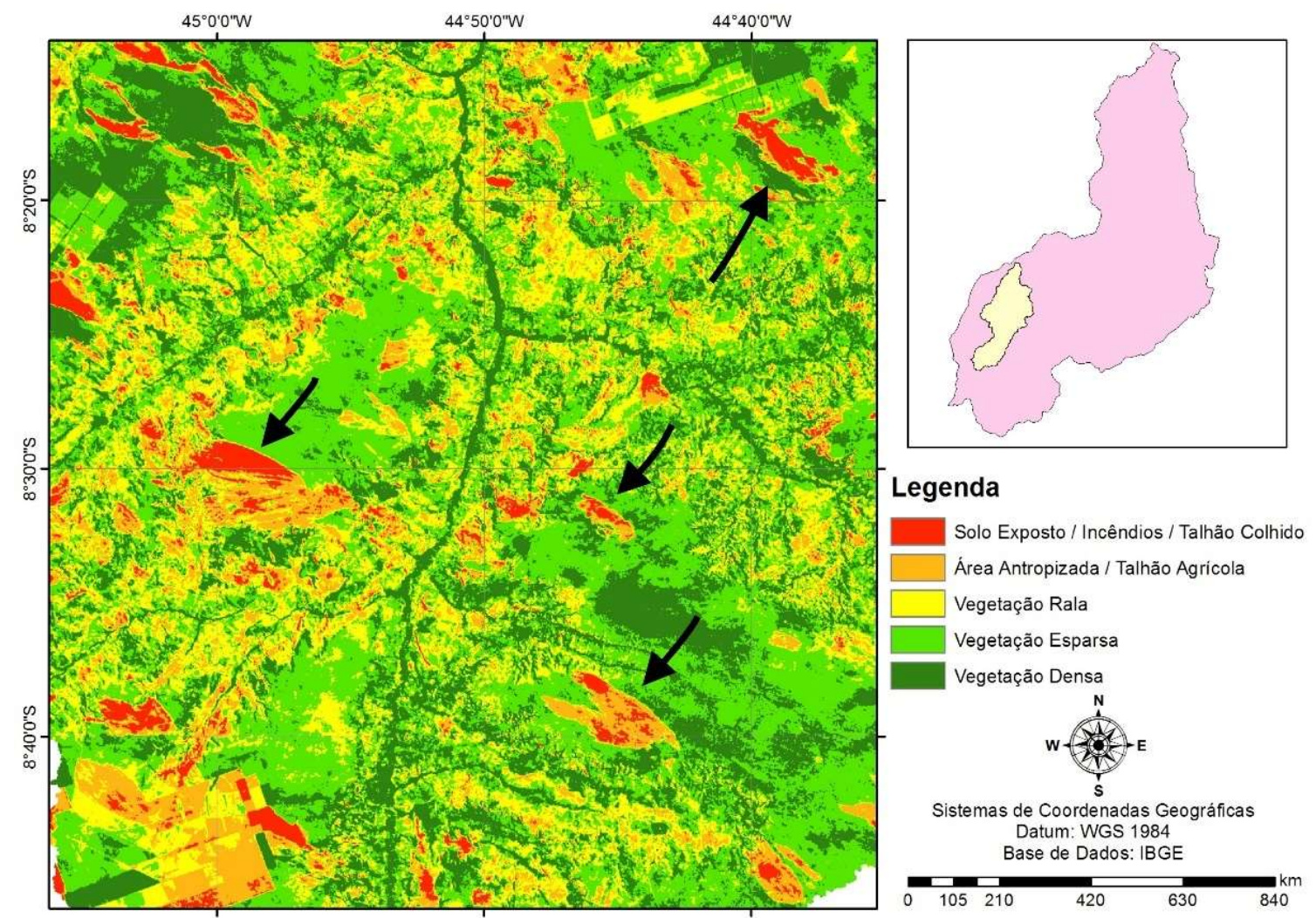

Figura 3. Indicação de áreas (setas pretas) que sofreram incêndios florestais na década de 1990.

Figure 3. Indication of areas (black arrows) that suffered forest fires in the 1990.

\section{DISCUSSÃO}

Ao longo dos anos, o Cerrado vem passando por um rápido processo de intensificação e uso da terra, principalmente para fins de cultivos agrícolas e atividades agropecuárias. $\mathrm{Na}$ bacia hidrográfica do rio Uruçuí-Preto, as áreas com alta exploração entre o período de 1984 a 2015 mostraram ocorrência de uso intensivo da terra em toda sua extensão e consequente aumento em fragmentação da vegetação nativa, o que reflete negativamente na conservação da biodiversidade local. Santos et al. (2016) destacam que as mudanças da paisagem podem resultar em alterações na biologia, diversidade e vulnerabilidade ecológica dos fragmentos florestais, evidenciada pela intensificação do processo de fragmentação. 
Tabela 2. Evolução da cobertura vegetal da Bacia do Rio Uruçuí-Preto entre 1984 a 2015.

Table 2. Evolution of the vegetation cover of the Uruçuí-Preto River Basin between 1984 and 2015.

\begin{tabular}{ccccccc}
\multicolumn{7}{c}{ Evolução Temporal (1984-2015) } \\
\hline Classes / Ano & $\begin{array}{c}1984 \\
\left(\mathrm{Km}^{2}\right)\end{array}$ & $\begin{array}{c}1990 \\
\left(\mathrm{Km}^{2}\right)\end{array}$ & $\begin{array}{c}1999 \\
\left(\mathrm{Km}^{2}\right)\end{array}$ & $\begin{array}{c}2005 \\
\left(\mathrm{Km}^{2}\right)\end{array}$ & $\begin{array}{c}2010 \\
\left(\mathrm{Km}^{2}\right)\end{array}$ & $\begin{array}{c}2015 \\
\left(\mathrm{Km}^{2}\right)\end{array}$ \\
\hline Solo Exposto & 390,3 & 501,4 & 395,8 & 921,4 & $1.182,9$ & $1.498,20$ \\
Área Antropizada & $1.449,9$ & $1.485,5$ & $1.324,8$ & $1.639,9$ & $1.995,2$ & $2.145,80$ \\
Vegetação Rala & $2.038,5$ & $3.106,6$ & $3.930,1$ & $3.246,5$ & $4.003,2$ & $2.210,00$ \\
Vegetação Esparsa & $4.325,0$ & $6.511,6$ & $7.253,7$ & $7.571,2$ & $6.726,9$ & $6.435,60$ \\
Vegetação Densa & $7.743,2$ & $4.341,9$ & $3.042,5$ & $2.567,9$ & $1.999,4$ & $3.487,40$ \\
\hline
\end{tabular}

\section{DISCUSSÃO}

Ao longo dos anos, o Cerrado vem passando por um rápido processo de intensificação e uso da terra, principalmente para fins de cultivos agrícolas e atividades agropecuárias. Na bacia hidrográfica do rio Uruçuí-Preto, as áreas com alta exploração entre o período de 1984 a 2015 mostraram ocorrência de uso intensivo da terra em toda sua extensão e consequente aumento em fragmentação da vegetação nativa, o que reflete negativamente na conservação da biodiversidade local. Santos et al. (2016) destacam que as mudanças da paisagem podem resultar em alterações na biologia, diversidade $e$ vulnerabilidade ecológica dos fragmentos florestais, evidenciada pela intensificação do processo de fragmentação.

Os resultados aqui evidenciados para o aumento da perda da cobertura vegetal original, são apoiados por estudo desenvolvido por Matricardi et al. (2018) que desenvolveram modelagem do desmatamento para a região do MATOPIBA, com a construção e simulação de três cenários, sendo eles: tendencial, otimista e pessimista, onde assumindo-se qualquer um dos cenários do estudo, os desmatamentos previstos para o período analisado (2011 a 2050), poderão alcançar taxas consideradas altas, tais mudanças previstas na vegetação natural da região podem trazer complicações ambientais, com efeitos diversos e imprevisíveis. Os autores destacam ainda que aumento da fragmentação da vegetação nativa, com consequências para à formação dos corredores ecológicos e à biodiversidade na região, além dos potenciais efeitos na regulação dos recursos hídricos e nas mudanças climáticas.

$\mathrm{O}$ aumento do desflorestamento aqui evidenciado encontra-se em acordo com Noojipady et al. (2017) em verificação das taxas de desmatamento no Cerrado brasileiro, identificaram elevada expansão das terras cultiváveis no MATOPIBA entre 2003 e 2013, principalmente no Maranhão (51\%) e Piauí (46\%), apresentando aumento na conversão de florestas para usos agrícolas, mesmo quando a expansão de terras agrícolas mostrou uma relativa diminuição em outras regiões do Cerrado.

As áreas de vegetação densa diminuíram progressivamente com aumento expressivo de áreas de solo exposto e antropização, corroborando com o estudo de Barbosa et al. (2012) que enfatizam que o processo de uso e ocupação do terreno tem sido intensificado pelo surgimento de novos projetos agrícolas, os quais ocasionam intenso desmatamento de grandes áreas. Vieira et al. (2016) alertam ainda que, mudanças no uso e na cobertura do terreno podem provocar degradação em decorrência da perda de solo proveniente do arraste das partículas, resultando no assoreamento dos rios, o que causam danos muitas vezes irreversíveis.

Em estudo desenvolvido em uma das principais sub-bacias do rio Uruçuí-Preto, Silva et al. (2015) constataram avanço de $30,01 \%$ do desmatamento nas áreas de Cerrado, e redução de $24,60 \%$ de todas as áreas de mata nativa da sub-bacia hidrográfica riacho da Estiva entre 1984 e 2010 . Beuchle et al. (2015) em investigação realizada para o Cerrado de todo território nacional, constataram que o domínio apresentou entre 1990 a 2010, relativas perdas e ganhos de cobertura de árvores e outras formas de terras arborizadas, no entanto, com contínua perda líquida de vegetação natural observada para o bioma no período. Ressalta-se, assim, a necessidade do aproveitamento das terras com planejamento adequado de uso, manejo sustentável do solo e gestão ambiental, para que haja conservação dos recursos naturais e da biodiversidade.

Mazzetto Silva (2009) faz destaque ao fato de que o Cerrado brasileiro é uma grande região biogeográfica de extrema riqueza sociocultural e ecológica, e tal riqueza vem sendo ignorada pelo avanço da fronteira agrícola, subordinada, atualmente, ao agronegócio global das commodities.

No contexto da avaliação da mudança espaço-temporal da cobertura vegetal na bacia hidrográfica analisada, o uso do IVDN mostrou-se eficaz para os devidos fins e objetivo estabelecidos. Silveira et al. (2008) em estudo com uso de distintos índices de vegetação para caracterização de dinâmica sazonal do Cerrado, reforçaram que o melhor índice para o mapeamento das fisionomias, foi o IVDN.

Destaca-se neste estudo, que devido ao uso de diferentes sensores (Landsat 5 e 8), é necessário considerar-se que as imagens apresentam diferenças na resolução radiométrica, o que pode neste caso, afetar no resultado do ano $2015 \mathrm{em}$ relação aos demais anos avaliados. Schechi et al., (2014) enfatiza que uso de imagens digitais de baixa resolução, tais como Landsat 5 é apropriado para este tipo de mapeamento de grandes áreas, além de ser uma tecnologia acessível e economicamente viável.

\section{CONCLUSÕES}

Os resultados obtidos indicaram que as ferramentas de SIG e Sensoriamento Remoto contribuíram para a satisfatória geração dos mapas, classificação e interpretação das feições de cobertura vegetal na sua organização espaço-temporal para a bacia do rio Uruçuí-Preto. É possível inferir que a expansão acelerada das atividades agrícolas na bacia hidrográfica analisada, tem relação com a consequente redução da vegetação nativa local, observados as taxas de antropização, solo exposto e redução da cobertura vegetal no intervalo temporal avaliado ao longo de 31 anos.

Estes resultados podem auxiliar na elaboração de novos estudos científicos, bem como em estratégias para conservação do Cerrado e manejo adequado dos recursos naturais.

\section{REFERÊNCIAS}

ARSANJANI, J. J. Characterizing, monitoring, and simulating land cover dynamics using GlobeLand30: A case study from 2000 to 2030. Journal of Environmental 
Management, Leuven, v. 214, p. 66-75, 2018. DOI: https://doi.org/10.1016/j.jenvman.2018.02.090

BARBOSA, F.M.A.; COSTA, J.J.; SOUZA, M.R. A expansão dos fronts agrícolas e planejamento ambiental e territorial no nordeste brasileiro. Revista Geonorte, v. 3, n.4, p.379389, 2012. Disponível em: http://www.periodicos.ufam.edu.br/revistageonorte/article /view/1954

BEHERA, M. D.; TRIPATHI, P.; DAS, P.; SRIVASTAVA, S. K.; ROY, S. K.; JOSHI, C.; BEHERA, P. R.; DEKA, J.; KUMAR, P.; KHAN, M. L.; TRIPATHI, O. P.; DASH, T.; KRISHNAMURTHY, Y. V. N. Remote sensing based deforestation analysis in Mahanadi and Brahmaputra river basin in India since 1985. Journal of Environmental Management, Leuven, v. 206, p. 1192-1203, 2018. DOI: https://doi.org/10.1016/j.jenvman.2017.10.015

BEUCHLE, R.; GRECCHI, R. C.; SHIMABUKURO, Y. E.; SELIGER, R.; EVA, H. D.; SANO, E.; ACHARD, F. Land cover changes in the Brazilian Cerrado and Caatinga biomes from 1990 to 2010 based on a systematic remote sensing sampling approach. Applied Geography, Kennesaw, n. 58, p. 116-127, 2015. DOI: http://dx.doi.org/10.1016/j.apgeog.2015.01.017

BUAINAIN, A. M.; GARCIA, J. R.; FILHO, J. E. R. V. Dinâmica da Economia e da Agropecuária no MATOPIBA. Rio de Janeiro: IPEA - Instituto de Pesquisa Econômica Aplicada, 2017. 64 p. (Texto para Discussão, 2283)

COSTA, A. R; SANTOS, O. Expansão Agrícola e Vulnerabilidade Natural do Meio Físico no Sul Goiano. Revista Geografia em Atos, Presidente Prudente, n. 10, v. 2, p. 23-35, 2010.

ENVIRONMENTAL SYSTEMS RESEARCH INSTITUTE (ESRI). ArcGIS for Desktop. Versão. 10.3. Redlands: ESRI, 2013.

DANTAS, K. P.; MONTEIRO, M. S. L. Valoração dos Efeitos Internos da Erosão: Impactos da Produção de Soja no Cerrado Piauiense. Revista Economia e Sociologia Rural, Pato Branco, v. 48, n. 4, p. 619-633, 2010. DOI: http://dx.doi.org/10.1590/S0103-20032010000400006

EL-ZEINY, A.; EFFAT, H. A. Environmental monitoring of spatiotemporal change in land use/land cover and its impacto $\mathrm{n}$ land surface temperature in El-Fayoum governorate, Egypt. Remote Sensing Applications: Society and Environment, Hong Kong, v. 8, p. 266-277, 2018. DOI: http://dx.doi.org/10.1016/j.rsase.2017.10.003

FRANÇA, L. C. J.; OLIVEIRA, R. J.; RIBEIRO, N. M. R.; SANTOS, E. L.; NORONHA, F. C. C.; RIBEIRO, A. T. Caracterização da cobertura vegetal e uso do solo no município de Uruçuí, Piauí, Brasil. Nativa, Sinop, v. 5, n. 5, p. 33-341, 2017. DOI: http://dx.doi.org/10.5935/23187670.v05n05a06

IBGE - INSTITUTO BRASILEIRO DE GEOGRAFIA E ESTATÍSTICA. Sistema IBGE de Recuperação Automática (Sidra). Rio de Janeiro: IBGE, 2016b. Disponível em: https://sidra.ibge.gov.br/home/ipp/brasil. Acesso em: 27 de janeiro 2018.

JÚNIOR, C. A. S.; LIMA, M. Soil Moratorium in Mato Grosso: Deforestation undermines the agreement. Land Use Policy, Adelaide, v. 71, p. 540-542, 2018. DOI: https://doi.org/10.1016/j.landusepol.2017.11.011

JUNIOR, C. A. S.; COUTINHO, A. D.; OLIVEIRA-JUNIOR, J. F.; TEODORO, P. E.; LIMA, M.; SHAKIR, M.; GOIS,
G.; JOHANN, J. A. Analysis of the impact on vegetation caused by abrupt deforestation via orbital sensor in the environmental disaster of Mariana, Brazil. Land Use Policy, Adelaide, v. 76, p. 10-20, 2018. DOI: https://doi.org/10.1016/j.landusepol.2018.04.019

LIMA, T. P.; FRANÇA, L. C. J.; SILVA, J. B. L.; FURTINI, M. B. Intensidade de Exploração do Solo em uma SubBacia do Rio Uruçuí-Preto, Piauí. Revista Educação Agrícola Superior, Brasília, v. 29, n. 1, 2014. DOI: http://dx.doi.org/10.12722/0101-756X.v29n01a02

LUSTIG, A.; STOUFFER, D. B.; ROIGÉ, M.; WORNER, S. P. Towards more predictable and consistent landscape metrics across spatial scales. Ecological Indicators, Coimbra, v. 57, p. 11-21. DOI: http://dx.doi.org/10.1016/j.ecolind.2015.03.042

MATRICARDI, E. A. T.; AGUIAR, A. S.; MIGUEL, E. P.; ANGELO, H.; GASPAR, R. O. Modelagem do desmatamento na região do MATOPIBA. Nativa, Sinop, v. 6 , n. 2 , p. 198-206, 2018. DOI: http://dx.doi.org/10.31413/nativa.v6i2.5092

MAZZETTO SILVA, C. E. Ordenamento Territorial no Cerrado brasileiro: da fronteira monocultora a modelos baseados na sociobiodiversidade. Desenvolvimento e meio ambiente, Curitiba, n. 19, p. 89-109, 2009. DOI: http://dx.doi.org/10.5380/dma.v19i0.16407

MEDEIROS, M. B. Manejo de fogo em Unidades de Conservação do Cerrado. Boletim do Herbário Ezechias Paulo Heringer, v. 10, p. 75 - 88, 2002.

MYERS, N.; MITTERMEIER, R. A.; MITTERMEIER, C. G.; FONSECA, C. G. A. B..; Kent, J. Biodiversity hotspots for conservation priorities. Nature, Londres, v. 403, p. 853858, 2000. https://dx.doi.org/10.1038/35002501

NASCIMENTO, T. V.; FERNANDES, L. L. Mapeamento de uso e ocupação do solo em uma pequena bacia hidrográfica da Amazônia. Ciência e Natura, Santa Maria, v. 39, n. 1, p. 170-178, 2017. DOI: http://dx.doi.org/10.5902/2179460X21737

NOOJIPADY, P.; MORTON, D. C.; MACEDO, M. N.; VICTORIA, D. C.; CHENGQUAN, H.; GIBBS, H. K.; BOLFE, E. L. Forest carbono emissions from cropland expansion in the Brazilian Cerrado biome. Environmental Research Letters, Berkeley, v. 12, n. 2, p. 2-11, 2017. DOI: https://dx.doi.org/10.1088/1748-9326/aa5986

PONZONI, F.J.; SHIMABUKURO, Y. E.; KUPLICH, T. M. Sensoriamento remoto da vegetação. São Paulo: Oficina de Textos, 2015. 165 p.

REYDON, B. P.; MONTEIRO, M. S. L. A ocupação do cerrado piauiense: um processo de valorização fundiária. REYDON, B. P.; CORNÉLIO; F.N.M (Orgs). Mercados de terras no Brasil: estrutura e dinâmica. Brasília: NEAD, 2006. p. 95-122.

RIBEIRO, J. F.; WALTER, B. M. T. Fitofisionomias do bioma Cerrado. In: SANO, S. M.; ALMEIDA, S. P. de. (Ed.). Cerrado: ambiente e flora. Planaltina: Embrapa/CPAC, 1998. p. 89-166.

RODRIGUEZ, M. P. R.; SOARES, R. V. Análisis comparativo entre los incendios forestales en Monte Alegre, Brasil y Pinar del Río, Cuba. Floresta, Curitiba, v. 34 , n. 2, p. 101-107, 2004. DOI: http://dx.doi.org/10.5380/rf.v34i2.2379

SAITO, N. S.; ARGUELLO, F. V. P.; MOREIRA, M. A.; SANTOS, A. R.; EUGENIO, F. C.; FIGUEIREDO, A. C. Uso da geotecnologia para análise temporal da cobertura 
florestal. Cerne, Lavras, v. 22, n. 1, p. 11-18, 2016. DOI: http://dx.doi.org/10.1590/01047760201622011935

SANTOS, A. R.; RIBEIRO, C. A. S.; PELUZIO, T. M. O.; PELUZIO, J. B. E.; QUEIROZ, V. T.; BRANCO, E. R. F.; LOREZON, A. S.; DOMINGUES, G. F.; MARCATTI, G. E.; CASTRO, N. L. M.; TEIXEIRA, T. R.; SANTOS, G. M. A. D. A.; MOTA, P. H. S.; SILVA, S. F.; VARGAS, R.; CARVALHO, J. R.; MACEDO, L. L.; ARAÚJO, C. S.; ALMEIDA, S. L. H. Geotechonology and landscape eecology applied to the selection of potential Forest fragments for seed harvesting. Journal of Environmental Management, Leuven, v. 183, p. 1050-1063, 2016. DOI: https://doi.org/10.1016/j.jenvman.2016.09.073

SAVAGE, S. L.; LAWRENCE, R. L.; SQUIRES, J. R. Mapping post-disturbance forest landscape composition with Landsat satellite imagery. Forest Ecology and Management, Arizona, v. 399, p. 9-23, 2017. DOI: https://doi.org/10.1016/j.foreco.2017.05.017

SCHECHI, R. G.; BATISTA, M. P.; BIACHI, R. C.; GUIMARÃES, R. Z.; RIZZI, N. E. Occupation Evolution on pequeno river watershed - São José dos Pinhaes - PR over 30 years. Nativa, Sinop, v. 2, n. 3, p. 149-154, 2014. DOI: http://dx.doi.org/10.14583/2318-7670.v02n03a04

SILVA, J. B. L.; SOUSA, P. A.; SOUZA, K. B.; ALMEIRA, K. N. S.; PIRE, L. C. Evolução Temporal do Desmatamento na Bacia do Riacho da Estiva, Piauí. Engenharia na Agricultura, Viçosa, v. 23, n. 4, p. 363370, 2015.
SILVEIRA, E. M. O.; CARVALHO, L. M. T.; ACERBIJÚNIOR, F. W.; MELLO, J. M. The assessment of vegetation seasonal dynamics using multitemporal NDVI and EVI images derived from MODIS. Cerne, Lavras, v. 14, n. 2, p. 177-184, 2008.

SOUSA, J. R. L.; BOTREL, R. T.; ALVES, A. R. Ocorrência de incêndios florestais na região de Bom Jesus, Sul do Estado do Piauí. Scientia Plena, Aracajú, v. 8, n. 4, 2012.

VALJAREVIC, A; DJEKIC, T.; STEVANOVIC, V.; IVANOVIC, R.; JANDZIKOVIC, B. GIS numerical and remote sensing analyses of forest changes in the Toplica region for the period of 1953-2013. Applied Geography, Kennesaw, v. 92, p. 131-139, 2018. DOI: https://doi.org/10.1016/j.apgeog.2018.01.016

VIEIRA, N. P. A.; SILVA, F. B.; NASCIMENTO, C. R.; VIEIRA, E. O.; FRAGA, M.S. Soil losses in the Cedro river basin/MG based on the sensor TM/LANDSAT-5 images. Nativa, Sinop, v. 4, n. 5, p. 261-270, 2016. DOI: http://dx.doi.org/10.14583/2318-7670.v04n05a01

WANG, J.; LIN, Y.; GLENDINNING, A.; XU, Y. Land-use changes and land policies evolution in China's urbanization processes. Land Use Policy, Adelaide, v. 75, p. 375-387, $2018 . \quad$ DOI: https://dx.doi.org/10.1016/j.landusepol.2018.04.011

WU, J.; GAO, Z.; LIU, Q.; LI, Z.; ZHONG, B. Methods for sandy land detection based on multispectral remote sensing data. Geoderma, Wagningen, v. 316, p. 89-99, 2018.

DOI: 